\title{
GENERIC STABILITY OF THE SPECTRA FOR BOUNDED LINEAR OPERATORS ON BANACH SPACES
}

\author{
LUO QUN \\ Guizhou Normal University \\ Department of Mathematics \\ Guiyang, PR of China 550001
}

(Received January, 1997; Revised August, 1998)

In this paper, we study the stability of the spectra of bounded linear operators $B(X)$ in a Banach space $X$, and obtain that their spectra are stable on a dense residual subset of $B(X)$.

Key words: Bounded Linear Operator, Spectra, Usco Mapping, Essential.

AMS subject classifications: $47 \mathrm{~A} 10$.

\section{Introduction}

Spectral theory is an important part of functional analysis, which attracted many authors, e.g. [1, 3]. It is known (see Kreyszig [3]) that the spectra of a bounded linear operator is a nonempty compact subset of complex plane $C$. When the operator is perturbed, how does its spectrum change? After Rayleigh and Schrödinger created perturbation theory, stability of spectra has been intensively developed. In a finitedimensional space, the eigenvalues of a linear operator $T$ depend on $T$ continuous [1], but it does not apply to a general Banach space. Kato [1, pp. 210] gives an example, in which he shows that the set of spectrum of a bounded linear operator in Banach space is not stable.

In this paper, by using Lemma 2.3 of K.K. Tan, J. Yu, and X.Z. Yuan [4], we obtain that the spectra of a bounded linear operator is stable on a dense residual subset of $B(X)$.

\section{Preliminaries}

If $X$ and $Y$ are two topological spaces, we shall denote by $K(X)$ and $P_{0}(Y)$ the space of all nonempty compact subsets of $X$ and the space all nonempty subsets of $Y$, respectively, both endowed with the Vietoris topology (see Klein and Thompson [2]). Then a mapping $T: X \rightarrow P_{0}()$ is said to be $(i)$ upper (resp. lower) semicontinuous at 
$x \in X$ if, for each open set $G$ in $Y$ with $G \supset T(x)$ (resp. $G \cap T(x) \neq \emptyset$ ), there exists an open neighborhood $O(x)$ of $x$ in $X$ such that $G \supset T\left(x^{\prime}\right)$ (resp. $G \cap T\left(x^{\prime}\right) \neq \emptyset$ ) for each $x^{\prime} \in O(x)$; (ii) $T$ is upper (resp. lower) semicontinuous on $X$ if, $T$ is upper (resp. lower) semicontinuous at each $x \in X$; (iii) $T$ is an usco mapping if, $T$ is upper semicontinuous with nonempty compact values.

The following result is due to K.K. Tan, J. Yu, and X.Z. Yuan [4, Lemma 2.3].

Theorem 2.1: If $X$ is (completely) metrizable, $Y$ is a Baire space and $T: Y \rightarrow$ $K(X)$ is a usco mapping, then the set of points where $T$ is lower semicontinuous is a (dense) residual set in $Y$.

Let $C$ denotes the whole complex plane, $X$ denotes a complex Banach space, $T$ : $X \rightarrow X$ linear operator. Then, $\sigma(T)=\{\lambda \in C: T-\lambda I$ is not invertible $\}$ is called the spectra of $T$, the complementary set $\rho(T)=C \backslash \sigma(T)$ is called the resolvent set of $T$. Here $I$ is the identity mapping.

The following theorems are from Kreyszig [3].

Theorem 2.2: Let $X$ and $Y$ be complex (or real) topological vector spaces, $T$ : $D(T) \rightarrow Y$ be a linear operator, and $D(T) \subset X, R(T) \subset Y$. Then

(1) $T^{-1}: R(T) \rightarrow D(T)$ exists if and only if $T x=0$ implies that $x=0$;

(2) if $T^{-1}$ exists, then $T^{-1}$ is a linear operator.

Theorem 2.3: The spectra $\sigma(T)$ of a bounded linear operator $T$ in Banach space is a nonempty compact subset of $C$.

Let $B(X, Y)$ be the set of all bounded linear operators from $X$ to $Y$ and let $C O(X, Y)$ be the set of closed linear operators from $X$ to $Y$.

The following theorem is due to T. Kato [1, Theorem 2.23].

Theorem 2.4: Let $T, T_{n} \in C O(X, Y), n=1,2, \ldots$,

(1) if $T \in B(X, Y)$, then $T_{n} \rightarrow T$ in the generalized sense if and only if $T_{n} \in$ $B(X, Y)$ for sufficiently large $n$ and $\left\|T_{n}-T\right\| \rightarrow 0$;

(2) if $T^{-1}$ exists and belongs to $B(X, Y)$, then $T_{n} \rightarrow T$ in the generalized sense if and only if $T_{n}^{-1}$ exists and belongs to $B(X, Y)$ for sufficiently large $n$ and $\left\|T_{n}^{-1}-T^{-1}\right\| \rightarrow 0$.

\section{Main Results}

Let $X \neq\{0\}$ be a complex Banach space, and let $B(X)$ denote the set of all bounded linear operators in $X$. Then $B(X)$ is a Banach space.

Theorem 3.1: $\sigma: B(X) \rightarrow K(C)$ is an usco mapping.

Proof: By Theorem 2.3, $\sigma(T)$ is a nonempty compact subset of $C$ for each $T \in B(X)$. Suppose that $\sigma$ is not upper semicontinuous at some $T_{0} \in B(X)$, i.e., that for any $\varepsilon_{0}>0$ there is a $\delta>0$, such that for all $S \in B(X)$ with $\left\|X-T_{0}\right\|<\delta$,

$$
H_{+}\left(\sigma\left(T_{0}\right), \sigma(S)\right)=\sup _{\lambda \in(S)}\left\{\operatorname{dist}\left(\lambda, \sigma\left(T_{0}\right)\right)\right\} \geq \varepsilon_{0}
$$

where $H$ is the Hausdorff metric and $H(\cdot, \cdot)=\max \left\{H_{+}(\cdot, \cdot), H_{-}(\cdot, \cdot)\right\}$. Then there exists a $\lambda_{0} \in \sigma(S)$ such that $\operatorname{dist}\left(\lambda_{0}, \sigma\left(T_{0}\right)\right) \geq \varepsilon_{0}>0$. Thus

$$
\lambda_{0} \notin \sigma\left(T_{0}\right), \quad \lambda_{0} \in \rho\left(T_{0}\right), \quad\left(T_{0}-\lambda_{0} I\right)^{-1} \in B(X),
$$

and, by Theorem 2.4 , 


$$
\left(S-\lambda_{0} I\right)^{-1} \in B(X), \lambda_{0} \notin \sigma(S),
$$

which contradicts that $\lambda_{0} \in \sigma(S)$. Therefore, $\sigma$ is an usco mapping.

Definition 3.1: For each $T \in B(X)$,

(i) $\quad \lambda \in(T)$ is an essential spectrum value relative to $B(X)$ if, for each open neighborhood $N(\lambda)$ of $\lambda$ in $C$, there exists an open neighborhood $O(T)$ of $T$ in $B(X)$ such that $\sigma\left(T^{\prime}\right) \cap N(\lambda) \neq \emptyset$ for each $T^{\prime} \in O(T)$;

(ii) $T$ is essential relative to $B(X)$ if, every $\lambda \in \sigma(T)$ is an essential spectrum value relative to $B(X)$.

Theorem 3.2: (1) $\sigma$ is lower semicontinuous at $T \in B(X)$ if and only if $T$ is essential relative to $B(X)$;

(2) $\sigma$ is continuous at $T \in B(X)$ if and only if $T$ is essential relative to $B(X)$.

Proof: (1) $\sigma$ is lower semicontinuous at $T \in B(X)$ if and only if each $\lambda \in \sigma(T)$ is an essential spectrum value relative to $B(X)$ and $T$ is essential relative to $B(X)$.

(2) The proof follows from (1) and Theorem 3.1.

Theorem 3.3: If $T \in B(X)$ such that $\sigma(T)$ is a singleton set, then $T$ is essential relative to $B(X)$.

Proof: Suppose $\sigma(T)=\{\lambda\}$, and let $G$ be any open set in $C$ such that $\sigma(T) \cap$ $G \neq \emptyset$. Then $\lambda \in G$, so that $\sigma(T) \subset G$. Since $\sigma$ is upper semicontinuous at $T$, by Theorem 3.1, there exists an open neighborhood $O(T)$ of $T$ in $B(X)$ such that $\sigma\left(T^{\prime}\right) \subset G$ for each $T^{\prime} \in O(T)$. In particular, $G \cap \sigma\left(T^{\prime}\right) \neq \emptyset$ for each $T^{\prime} \in O(T)$. Thus $\sigma$ is lower semicontinuous at $T$, and by Theorem $3.2(1), T$ is essential relative to $B(X)$.

Theorem 3.4: Let $C$ be complex plane, and $X \neq\{0\}$ be a complex Banach space. Then there exists a dense residual subset $Q$ of $B(X)$ such that $T$ is essential relative to $B(X)$ for each $T \in Q$.

Proof: By Theorem 3.1 and Theorem 2.1, $\sigma$ is lower semicontinuous on some dense residual subset $Q$ of $B(X)$. Consequently, by Theorem $3.2(1), T$ is essential relative to $B(X)$ for each $T \in Q$.

\section{References}

[1] Kato, T., Perturbation Theory for Linear Operator, Springer-Verlag, New York 1966.

[2] Klein, E. and Thompson, A., Theory of Correspondences, Wiley, New York 1984.

[3] Kreyszig, E., Introductory Functional Analysis with Applications, John Wiley \& Sons, Inc. 1978.

[4] Tan, K.K., Yu, J. and Yuan, X.Z., The stability of Ky Fan's points, Proc. AMS 123 (1995), 1511-1518. 


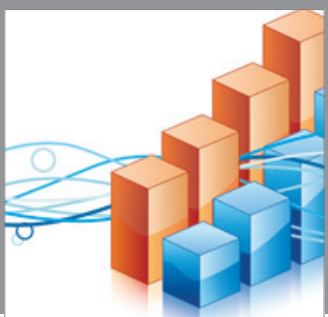

Advances in

Operations Research

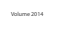

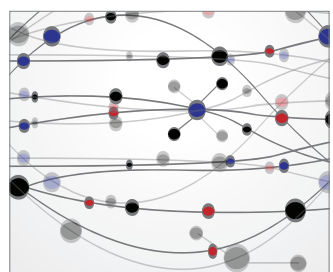

\section{The Scientific} World Journal
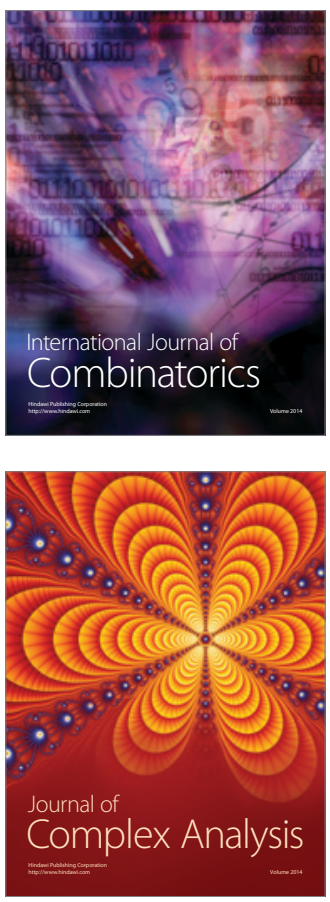

International Journal of

Mathematics and

Mathematical

Sciences
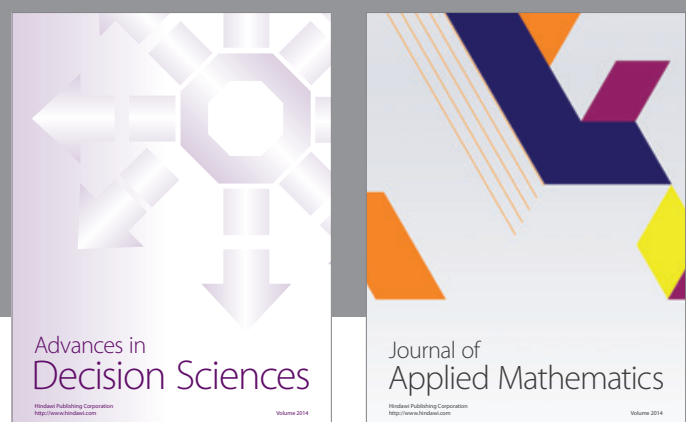

Journal of

Applied Mathematics
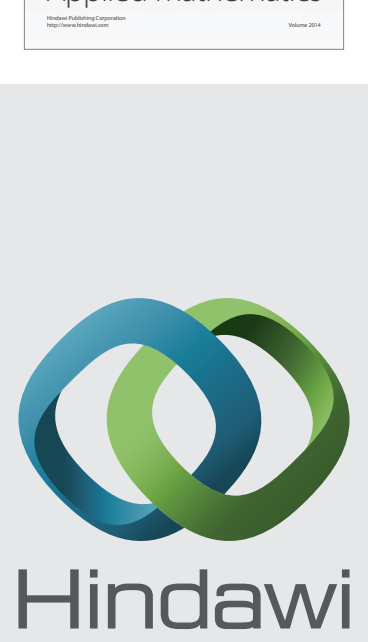

Submit your manuscripts at http://www.hindawi.com
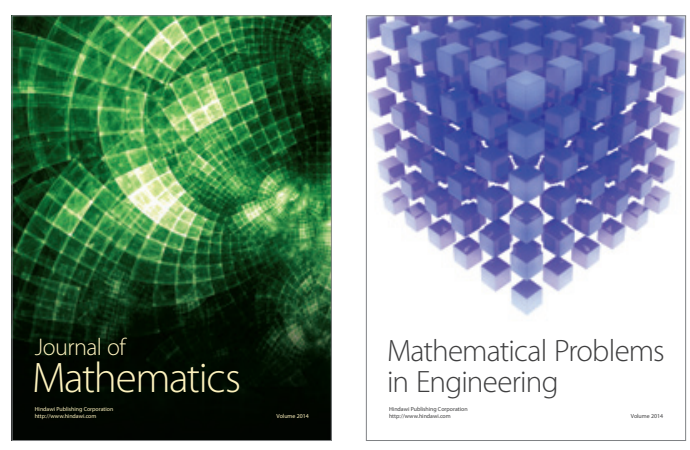

Mathematical Problems in Engineering
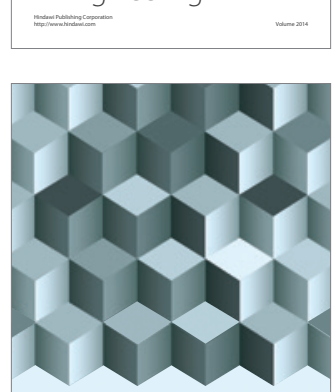

Journal of

Function Spaces
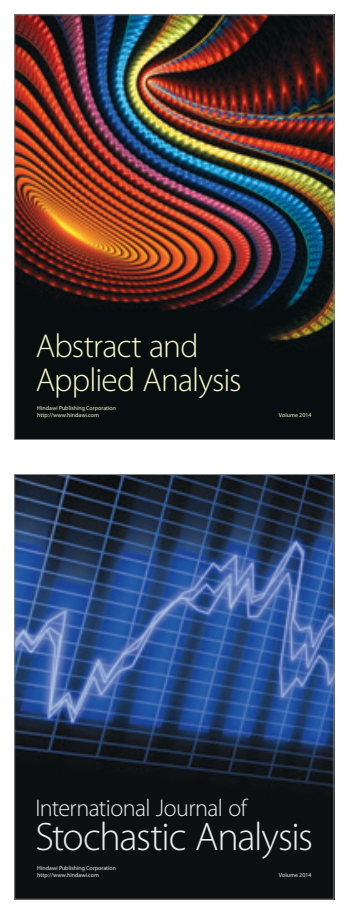

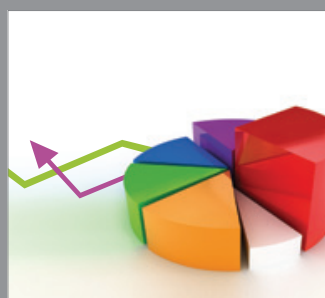

ournal of

Probability and Statistics

Promensencen
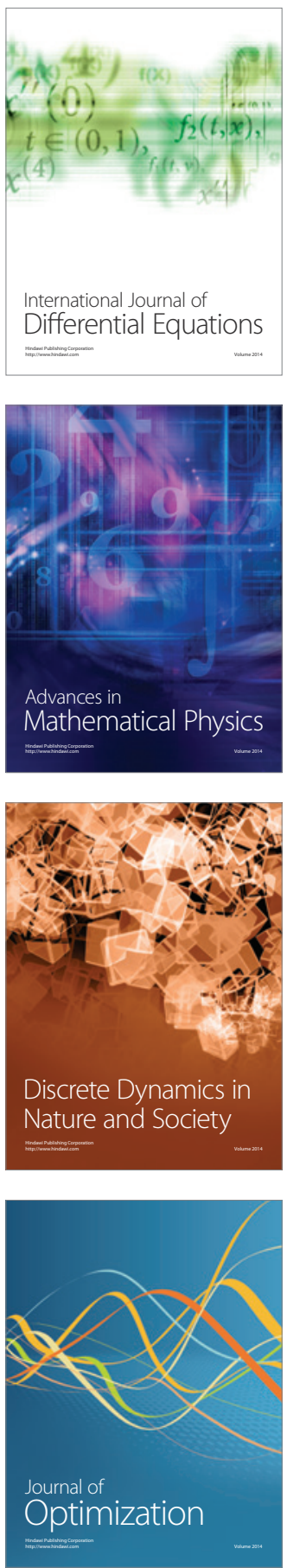